Einsichten aus der experimentellen Wirtschaftsforschung zur Tragik von Gemeingütern

\section{Kein unlösbares Dilemma}

\section{Probleme des Umweltschutzes werden häufig als Tragödie der Allmende be- schrieben. Dieses soziale Dilemma gründet in der Annahme strikt eigennützigen Verhaltens. Ökonomische Experimente zeigen aber in der Regel ein anderes Verhaltensmuster. Dies gilt selbst dann wenn zukünftige Generationen einbe- zogen werden. Die soziale Motivation kann durch geeignete Institutionen ver- stärkł werden.}

$\mathrm{U}$

Von Simon Gächter mweltressourcen wie saubere Luft, ökologisch stabile Gewässer, der Fischbestand in Seen und Meeren, die gemeinsame Bewirtschaftung von Viehweiden, die Nutzung von Waldbeständen, aber auch der Straßenverkehr haben eines gemeinsam: ihre unregulierte, individuelle Benutzung erzeugt ein soziales Dilemma. Jeder Benutzer von Umweltressourcen hat einen individuellen Anreiz, mehr Ressourcen zu verbrauchen, als es sozial wünschenswert wäre. Spätestens seit der bahnbrechenden Arbeit von Garrett Hardin (1968) mit dem sprechenden Titel „The Tragedy of the Commons" ist diese ernüchternde Einsicht sozialwissenschaftliches Allgemeingut (1). Hardin beschreibt das Problem anschaulich anhand der Nutzung einer Allmende, zu der jeder Bauer Zugang hat. Jede Kuh, die der Bauer auf die Weide treibt, frisst Gras und erzeugt dann Milch und gutes Fleisch. Aus diesem Grund hat der Bauer einen Anreiz, möglichst viele Kühe auf die Weide zu treiben. Dies gilt natürlich gleichermaßen für alle Bauern. Wenn allerdings alle ihre Kühe auf die Weide treiben, dann ist die Allmende bald abgegrast und die Kühe bekommen nur noch wenig zu fressen. Das soziale Dilemma besteht nun darin, dass alle Bauern individuell immer einen Anreiz haben, möglichst alle Kühe auf die Weide zu treiben, aber alle Bauern gemeinsam dann mit überweideten Allmenden leben müssen. Eine ähnliche Logik gilt für alle oben genannten Beispiele.

Man mag nun einwenden, dass die Bauern eines Dorfes wohl in der Lage sein werden, das soziale Dilemma zu erkennen und gemeinsam zu handeln, um diese Tragik der Allmende zu vermeiden. Schließlich liegt es ja im Gemeinschaftsinteresse der Bauern hier eine Lösung zu finden! Die Publikation des Buches ,The Logic of Collective Action" von Mancur Olson im Jahre 1965 hat allerdings diesem Argument den Boden unter den
Füßen weggezogen und zu einer weiteren Ernüchterung beigetragen (2). Erstens, nur weil umweltschonendes Verhalten im Gemeinschaftsinteresse liegt (was unbestritten ist), kann man daraus nicht schließen, dass es deshalb auch im Eigeninteresse jedes einzelnen ist, sich ökologisch zu verhalten. Und zweitens wies Olson darauf hin, dass auch kollektives Handeln eine soziale Dilemmastruktur aufweist: wenn sich zwei Bauern darauf einigen, weniger Kühe aufs Feld zu bringen, dann freut sich der Dritte. Unter der Annahme, dass die Akteure in ihrem Eigeninteresse handeln werden und in der Lage sind, geeignete Abmachungen zu treffen, bzw. Sanktionen zu setzen, können vielleicht einige wenige Bauern ihr soziales Dilemma vermeiden. In größeren Gruppen hingegen dominiert, so Olson, das Eigeninteresse und die Bauern werden dementsprechend ihr Übernutzungsproblem durch gemeinsames Handeln nur schwerlich überwinden können. Die moderne ökonomische Forschung hat diese Argumente durch allgemeine formale Modelle theoretisch untermauert.

\section{Auswege aus dem Dilemma}

Da die meisten Umweltnutzungsprobleme eine soziale Dilemma-Struktur aufweisen, scheinen die Aussichten für eine sozial verträgliche Nutzung von Umweltressourcen tatsächlich düster. Die Praxis sieht allerdings zum Glück nicht ganz so verhängnisvoll aus. Wie Elinor Ostrom in ihrem Buch „Governing the Commons" (1990) gezeigt hat, finden sich auf der ganzen Welt Beispiele für erfolgreiche Bewirtschaftungen von Umweltgemeingütern (3). Offensichtlich sind Menschen in der Lage, die soziale Dilemma-Struktur durch geeignete Institutionen zu überwinden. Andere Gemeingüter hingegen erleiden offensichtlich das Schicksal der Tragik der Allmende. Wie kann man das erklären? Es stellen sich dabei zwei Fragen: Erstens, welche Faktoren und Institutionen erwei- sen sich empirisch als geeignet, soziale DilemmaStrukturen zu überwinden? Zweitens, ist denn die Eigennutzannahme, die hinter den Argumenten von Hardin, Olson und vielen ökonomischen Modellen steht, wirklich gerechtfertigt? Oder ist es nicht vielmehr so, dass viele Menschen bereit sind, sich umweltbewusst zu verhalten, wenn andere dies auch tun, auch wenn es dem unmittelbaren Eigeninteresse widerspricht? Antworten auf diese Fragen sind von großer theoretischer und praktischer Bedeutung, aber anhand von konkreten Gemeingütern sehr schwer zu beantworten. Wenn man beispielsweise wissen will, wie eine andere Nutzungsregelung als die derzeit verwendete funktionieren würde, dann kann man in der Praxis nicht einfach die derzeitige Regelung durch eine andere ersetzen, um zu sehen, wie sie funktioniert. Noch schwieriger ist das Problem, wenn man die Frage nach der empirischen Bedeutung der strikten Eigennutzannahme beantworten will, weil man dazu die Präferenzen der Menschen kennen muss und diese im Feld schwer messbar sind.

Die experimentelle Wirtschaftsforschung, welche in diesem Jahr mit einem Nobelpreis gewürdigt worden ist (vgl. S. 10), stellt ein Instrument zur Verfügung, mit dessen Hilfe man einen Schritt weiter zur Beantwortung beider Fragen kommt. Der Experimentator kann im Labor die Spielregeln vorgeben und so die Versuchspersonen in eine soziale Dilemma-Situation versetzen. Ein typisches Experiment sieht beispielsweise folgendermaßen aus: Vier Versuchspersonen bilden eine Gruppe. Jeder erhält eine Ausstattung von $20 \mathrm{Eu}-$ ro. Diese 20 Euro kann die Versuchsperson nun für sich behalten, oder aber jeden beliebigen Betrag zwischen 0 und 20 Euro in ein Gemeinschaftsprojekt investieren. Die Gruppenmitglieder entscheiden anonym und gleichzeitig. Der Experimentator verdoppelt dann den Betrag, den alle vier ins Gemeinschaftsprojekt gezahlt haben und verteilt den verdoppelten Betrag gleichmäßig auf alle Gruppenmitglieder.

Dieses Spiel weist eine soziale Dilemma-Struktur auf: Wenn ich einen Euro in das Projekt einzahle, dann wird dieser Euro zwar verdoppelt, aber dann auf alle vier Gruppenmitglieder verteilt. Ich erhalte von meiner Investition nur 50 Cent zurück. Also werde ich meinen Euro nicht ins Projekt investieren. Wenn andere hingegen investieren, dann profitiere ich, auch wenn ich mich als Trittbrettfahrer verhalten und nichts beigetragen habe. Dieses Argument gilt für jeden Euro-Betrag und gleichermaßen für alle Gruppenmitglieder. 
Deshalb wird niemand etwas ins Projekt einzahlen und jeder wird mit 20 Euro nach Hause gehen. Wenn hingegen alle vier Gruppenmitglieder ihre gesamten 20 Euro in das Projekt einzahlen würden, dann würde jeder 40 Euro ([80x2]/4) einstreifen!

\section{- Ein aufschlussreiches Experiment}

Dieses Spiel wird in der experimentellen Wirtschaftsforschung sehr häufig untersucht, weil es sehr einfach ist und eine klare Dilemma-Struktur aufweist. So haben Ernst Fehr und ich dieses Experiment verwendet, um etwas über die Motivationsstruktur zu erfahren, die hinter dem Verhalten in sozialen Dilemma-Situationen stecken könnte. Wir haben dieses Experiment in zwei Versuchsanordnungen durchgeführt. Die erste Versuchsanordnung ist jene, die ich soeben beschrieben habe. Die Versuchspersonen waren 240 Studierende der Universität Zürich, die in Gruppen zu je vier Personen dieses Spiel sechs mal wiederholt gespielt haben. Dabei wurde den Versuchspersonen klar gemacht, dass in jeder der sechs Wiederholungen die Gruppenmitglieder vollständig ausgewechselt werden. Niemand spielt mehr als einmal mit denselben Gruppenmitgliedern. Deshalb gilt unter der Eigennutzannahme für jede der sechs Wiederholungen die düstere Prognose der Tragik der Allmende. In der zweiten Versuchsanordnung haben wir die Spielregeln leicht geändert. Nach jeder Runde erfuhren die derzeitigen Gruppenmitglieder die Beiträge der anderen Gruppenmitglieder und konnten dann zu eigenen Kosten die anderen Gruppenmitglieder bestrafen. Eine rationale und eigennützige Versuchsperson wird jedoch nie Kosten aufwenden, um andere zu sanktionieren. Mit anderen Worten, auch in der zweiten Versuchsanordnung sollten wir keine Beiträge zum Projekt beobachten. Allerdings sind vielleicht nicht alle Menschen eigennützig, sondern bereit, zum Projekt beizutragen, wenn andere dies auch tun. Jemand der in diesem Sinne bedingt kooperativ ist und außerdem nicht der Dumme sein will, der beiträgt, während die anderen schmarotzen, ist vielleicht trotz Kosten bereit, Trittbrettfahrer zu strafen. Alle Versuchspersonen nahmen an beiden Experimenten teil. Die Abbildung zeigt die Ergebnisse dieser beiden Versuche. Das Ergebnis des Experimentes ohne Bestrafungsmöglichkeiten bestätigt viele ähnliche Experimente, die vor uns durchgeführt worden sind. Im Durchschnitt tragen die Versuchspersonen klar mehr bei, als es mit der strikten Eigennutzhypothese verträglich ist, aber die Beiträge sind auch deutlich unterhalb des sozial optimalen Beitrags von 20. Im Verlauf des Experiments nimmt auch das Trittbrettfahrerverhalten immer mehr zu. Mit Hilfe von weiteren Experimenten haben wir herausgefunden, dass die Mischung von bedingt kooperativen Versuchspersonen und Trittbrettfahrern erklären kann, warum die Kooperation über die Zeit zusammenbricht: Die bedingt Kooperativen sind zwar anfänglich bereit, etwas beizutragen, stellen dann aber fest, dass es Trittbrettfahrer gibt und reduzieren demzufolge selbst ihre Beiträge, mit der Konsequenz, dass die Kooperation insgesamt erodiert und die Versuchspersonen im sozialen Dilemma gefangen sind.

Ganz anders sieht es nun in der Versuchanordnung mit Bestrafungsmöglichkeiten aus. Es zeigt sich, dass die Möglichkeit der individuellen Sanktionen sofort als glaubwürdig erachtet wird - die Beiträge sind bereits in der ersten Runde des neuen Experimentes höher als in der ersten Runde des Experimentes ohne Bestrafungsmöglichkeiten. Während im ersten Experiment die Beiträge unweigerlich fallen, tragen im zweiten Experiment dieselben Versuchspersonen im Durchschnitt immer mehr bei. Das Bestrafungsverhalten zeigt, warum dies so ist. Die Trittbrettfahrer wurden von denjenigen, die überdurchschnittlich investiert haben, bestraft und dies obwohl allen bewusst war, dass sie nie mehr gemeinsam in einer Gruppe sein werden! Offensichtlich ist die Verärgerung über die Schmarotzer groß genug, um diese zu eigenen Kosten zu disziplinieren.

\section{Bedingte Kooperation statt strik- tem Eigennutz}

Dieses und eine Reihe anderer verwandter Experimente (4) lehren uns zwei Dinge über die beiden oben gestellten Fragen. Erstens, die strikte Eigennutzannahme ist empirisch nicht haltbar. Viele Menschen sind bedingt kooperativ, aber es gibt auch Trittbrettfahrer. Das Experiment zeigt aber, zweitens, auch die Bedeutung von Institutionen. Allgemein gesprochen, legen Institutionen die Regeln fest, unter denen Menschen interagieren. Wenn die Interaktionen anonym sind und soziale Sanktionsmöglichkeiten nicht existieren, wie in unserem ersten Experiment, dann wird die Tragik der Allmende über kurz oder lang auftreten, und das obwohl nicht alle Menschen eigennützig sind.
Institutionelle Bedingungen, unter denen Trittbrettfahrer identifiziert und sanktioniert werden können, können allerdings die Tragik der Allmende substanziell vermindern. Dabei kann, wie die Experimente von Milinski, Semmann und Krambeck gezeigt haben (5), die Sanktion durchaus indirekt als ein Reputationsverlust auftreten, in dem andere mir in einem anderen Kontext nicht mehr helfen, wenn sie beobachten, dass ich nicht kooperiert habe.

\section{Und zukünftige Generationen?}

Diese Einsichten der experimentellen Forschung sind - komplementär zur Feld- und Institutionenforschung - wichtig, weil sie Erklärungen für funktionierende Allmenden geben und institutionelle Bedingungen aufzeigen, unter denen Kooperation erfolgreich sein kann (6). Doch wie ist es um die Interessen zukünftiger Generationen bestellt, die vom Zustand von Umweltgemeingütern beeinflusst sein werden, ohne dass sie gegenwärtig Handlungschancen haben? Die diskutierten Experimente können alle als Untersuchungen von Instrumenten verstanden werden, die es einer gegenwärtigen Generation erlauben, ihr gegenwärtiges Gemeingutproblem zu lösen. Reputation und reziprok motivierte Sanktionsbereitschaft, sowie geeignete Institutionen sind der Lösung von gegenwärtigen Gemeingutproblemen dienlich, weil die Kooperation mit gegenwärtigen „Mitspielern" erfolgt. Zukünftige Generationen sind naturgemäß nur passive Mitspieler - sie sind zwar direkt von den Folgen des Verhaltens der gegenwärtigen Generation beeinflusst, können selbst aber nicht deren Kooperationsverhalten beeinflussen. Ein zentraler Gesichtspunkt ist natürlich, wie schnell sich Ressourcen erneuern. Überweidete Wiesen wachsen sicherlich schneller nach als gerodete Waldbestände, wie im Amazonas zum Teil dramatisch sichtbar ist.
Abbildung 1: Kooperation mit und ohne Bestrafungsmöglichkeit

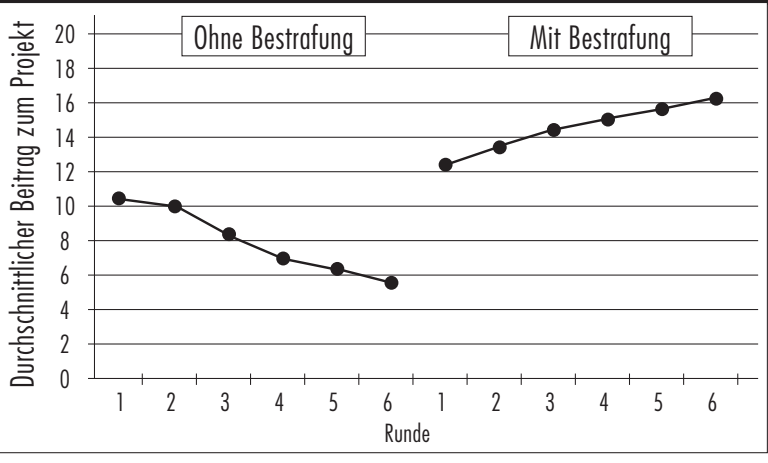

Quelle: Fehr, Ernst/Gächter, Simon:

Altruistic punishment in humans. In: Nature 415, 2002, S. 137-140 
Ein innovatives neues Experiment von Fischer, Irlenbusch und Sadrieb widmet sich der Frage, wie intergenerationale Dilemma-Probleme gelöst werden (7). Es gibt drei Versuchsanordnungen: ein Kontrollexperiment ohne zukünftige Generationen, in dem eine Variante des oben beschriebenen Spiels einmalig gespielt wird und zwei Hauptexperimente mit zukünftigen Generationen. Die beiden Hauptexperimente unterscheiden sich in bezug auf die Geschwindigkeit des Ressourcenwachstums. Es gibt eine schnell und eine langsam wachsende Ressource. Jede Generation entscheidet nur einmal und deren Gesamtergebnis bestimmt, nach dem entsprechenden Wachstum, den Ressourcenbestand, welcher der neuen Generation zur Verfügung steht. Eine Generation ist in diesem Experiment eine Gruppe von neuen Versuchspersonen, die über den bisherigen Verlauf des Experimentes nicht informiert ist und nur weiß, dass ihre Entscheidungen bei gegebener und bekannter Wachstumsregel den Ressourcenbestand für die zukünftige Generation von Versuchspersonen bestimmt.

Die Ergebnisse des Kontrollexperimentes bestätigen die bekannten Befunde: Die Kooperation ist relativ zum sozialen Optimum zu niedrig aber höher als es die Eigennutzthese prognostizieren würde. Die Hauptergebnisse allerdings sind ebenso dramatisch wie ernüchternd: bei lang- sam wachsenden Ressourcen, wo eine Ausbeutung des gegenwärtigen Ressourcenbestandes für die zukünftigen Generationen stärkere negative Konsequenzen hat als bei einer schnell wachsenden Ressource, ist der Anteil egoistischen Verhaltens höher als bei der schnell wachsenden Ressource! Dieses Ergebnis widerspricht einer Hypothese, die besagt, dass bei schwer erneuerbaren Ressourcen die Menschen ein stärkeres Gewicht auf Fairness und intergenerationale Gerechtigkeit legen als bei schnell wachsenden Ressourcen, wo die Interessen der zukünftigen Generationen auch weniger berührt sind. Der Grund für dieses überraschende Ergebnis ist, wie die Autoren herausgefunden haben, dass die Versuchspersonen bei der langsam wachsenden Ressource stärker als bei der schnell wachsenden Ressource glaubten, dass die anderen gegenwärtigen Versuchspersonen sich weniger egoistisch verhalten werden, weshalb sie selbst etwas eigennütziger waren.

Dieses Ergebnis ist natürlich nicht ohne weiteres auf die nachhaltige Bewirtschaftung von intergenerationalen Gemeingütern übertragbar. Ein weiteres Verständnis für soziale Motivationen in intergenerationalen Kooperationsproblemen ist nötig, um geeignete Maßnahmen ergreifen zu können. Die Methoden der experimentellen Wirtschaftsforschung stellen dabei ein wichtiges
Werkzeug zur Verfügung, das in der ökologischen Wirtschaftsforschung sicherlich noch ein starkes Wachstumspotenzial hat.

\section{Anmerkungen}

(1) Hardin, Garrett: The Tragedy of the Commons. In: Science 162 (1968), S. 1243-1248.

(2) Olson, Mancur: The Logic of Collective Action: Public Goods and the Theory of Groups. Cambridge MA 1965.

(3) Ostrom, Elinor: Governing the Commons. The Evolution of Institutions for Collective Action. Cambridge 1990.

(4) Vgl. z.B. Fehr, Ernst/ Gächter, Simon: Fairness and Retaliation: The Economics of Reciprocity. In: Journal of Economic Perspectives 14 (2000), Nr. 3, S. 159-181.

(5) Milinski, Manfred/Semmann, Dirk/ Krambeck, HansJürgen: Reputation helps solve the ,tragedy of the commons'. In: Nature 415 (2002), S. 424-426.

(6) Für weitere Befunde siehe Ostmann, Axel: Grenzen ökonomischer Anreize für Umweltgemeingüter. In: GAIA 7 (1998), Nr. 4, S. 286-295.

(7) Fischer, Maria-Elisabeth/ Irlenbusch, Bernd/ Sadrieh, Abdolkarim: An Intergenerational Common Pool Resource Experiment. Arbeitspapier, Universität Erfurt 2002.

\section{Der Autor}

Dr. Simon Gächter ist Professor für angewandte Mikroökonomik an der Universität St. Gallen.

Kontakt: FEW-HSG, Varnbüelstraße 14, CH-9000 St. Gallen. Tel. 0041-71-224-2535, Fax -2302,

E-Mail: simon.gaechter@unisg.ch

\section{Zug um Zug die Effizienz steigern}

\section{Hinter einer Reihe von Umweltproblemen steht eine Gemeingut-Problematik. Die Sozialpsychologie zeigt, wie Menschen mit den entstehenden Dilemmata umge- hen. Besondere Bedeutung für kooperative lösungen haben soziale Wertorientie- rungen, Sanktionen und Kontrollen sowie geeignete Reaktionsstrategien.}

$\mathrm{U}$ Von Martin Beckenkamp nsere heutige Zeit lässt sich trefflich als eine Ära der Umweltprobleme charakterisieren, zum Teil auch dadurch bedingt, dass die politische Wachsamkeit und Sensibilität gegenüber derartigen Problemen weltweit zugenommen hat. An der akademisch-wissenschaftlichen Auseinandersetzung mit dem Problem sind die unterschiedlichsten Disziplinen beteiligt. Von Beginn an waren die Naturwissenschaften und die Ingenieurwissenschaften eingebunden in Bestandsaufnahmen und die Entwicklung von Lösungsansä̈zen. In der Tat sind Umweltprobleme lange Zeit eher als tech- nische denn als sozialwissenschaftliche Probleme aufgefasst worden, doch mittlerweile werden auch Rechts-, Wirtschafts- und die Politikwissenschaften auf nationaler und internationaler Ebene herangezogen. In den letzten zehn bis fünfzehn Jahren hat sich sowohl bei nationalen Institutionen wie beispielsweise der VW Stiftung als auch bei internationalen Organisationen, wie etwa den Vereinten Nationen mit der von ihr beschlossenen Agenda 21, die Erkenntnis durchgesetzt, dass auch psychologische Problemstellungen hinter Umweltthemen stehen. Dennoch scheint ihr Stellenwert, verglichen mit den zuvor genannten Disziplinen, nicht sehr hoch. Zu Umweltproblemen kann die psychologische Forschung unterschiedliche Schwerpunkte setzen, die sich nicht gegenseitig ausschließen müssen: motivationspsychologische, kognitionspsychologische (vgl. hierzu den Beitrag von Beckenbach), und sozialpsychologische. Interessiert man sich für Umweltprobleme, hinter denen eine Gemeingut (common pool resources, CPR)-Problematik steht, sind insbesondere die sozialpsychologischen Beiträge relevant, während einige der Erkenntnisse aus der Kognitions- und Motivationspsychologie weit über diese Problematik hinausgehen und auch bei anders gearteten Umweltproblemen relevant sein können. In der Folge soll allein auf sozialpsychologische Ergebnisse fokussiert werden, die mit Hinblick auf CPRs relevant sein können.

\section{Soziale Dilemmata}

Nicht jedes Umweltproblem ist ein Gemeingutproblem, und umgekehrt resultiert es auch nicht zwingend, dass ein Gut, weil es ein Gemeingut ist, zu einem Umweltproblem führen muss. Die engsten Berührungspunkte speziell zur Gemein- 
(c) 20I0 Authors; licensee IÖW and oekom verlag. This is an article distributed under the terms of the Creative Commons Attribution Non-Commercial No Derivates License (http://creativecommons.org/licenses/by-nc-nd/3.o/), which permits unrestricted use, distribution, and reproduction in any medium, provided the original work is properly cited. 